\title{
Cancer of the pancreas: the best image for early detection - CT, MRI, PET or US?
}

\author{
Anthony E Hanbidge MB BCh FRCPC
}

AE Hanbidge. Cancer of the pancreas: The best image for early detection - CT, MRI, PET or US? Can J Gastroenterol 2002;16(2):101-105.

Pancreatic cancer has a poor prognosis, and the best chance for survival is to diagnose the tumour at an early stage. Abdominal ultrasound, computed tomography, magnetic resonance imaging and endoscopic retrograde cholangiopancreatography are the most commonly used radiological techniques for imaging the pancreas. The diagnostic evaluation should be tailored to the individual patient. Dual-phase helical computed tomography and magnetic resonance imaging have similar accuracies for detecting and staging pancreatic adenocarcinoma. Sonography results are highly dependent on the skill and persistence of the operator. No radiological examination is very sensitive at visualizing small metastases in the lymph nodes and peritoneum, or on the surface of the liver. Thus, it is difficult to establish with certainty whether a tumour is resectable. Another major challenge is to differentiate cancer from an inflammatory mass in chronic pancreatitis. Functional imaging (using positron emission tomography with fluorodeoxyglucose) may be helpful, especially if the images are fused with those of computed tomography or magnetic resonance imaging. The diagnostic accuracies, applications and limitations of the various modalities are discussed.

Key Words: Computed tomography; Magnetic resonance imaging; Pancreatic cancer; Positron emission tomography; Ultrasound

Résumé à la page suivante

Division of Abdominal Imaging, Joint Department of Medical Imaging, Mount Sinai Hospital and University Health Network, and Department of Medical Imaging, University of Toronto, Toronto, Ontario

Correspondence: Dr Anthony E Hanbidge, Division of Abdominal Imaging, University Health Network,

Toronto General Hospital, 200 Elizabeth Street, Toronto, Ontario M5G 2C4. Telephone 416-340-4800 ext 3291, fax 416-593-0502,

e-mail anthony.hanbidge@uhn.on.ca

Received for publication December 3, 2001. Accepted December 3, 2001 
Quelle est la meilleure technique de détection précoce du cancer du pancréas : la tomodensitométrie, l'imagerie par résonance magnétique, la tomographie par émission de positrons ou l'échographie?

RÉSUMÉ : Le cancer du pancréas comporte un sombre pronostic, et les meilleures chances de survie reposent sur le diagnostic précoce de la tumeur. L'échographie abdominale, la tomodensitométrie, l'imagerie par résonance magnétique (IRM) et la cholangio-pancréatographie rétrograde endoscopique constituent les techniques de radiologie les plus fréquemment utilisées pour visualiser le pancréas. L'évaluation diagnostique devrait être adaptée à chaque patient. L'IRM semble la technique la plus précise pour détecter la présence de masses dans le pancréas, tandis que la tomodensitométrie hélicoïdale biphasée pourrait fournir de l'information complémentaire sur l'invasion vasculaire. Les résultats de l'échographie dépendent en grande partie de la compétence et de la ténacité du technicien. Aucun examen radiologique n'est assez sensible pour permettre de visualiser les petites métastases présentes dans les ganglions lymphatiques et le péritoine ou à la surface du foie. Aussi est-il difficile de déterminer si une tumeur est résécable ou non. Une autre difficulté de taille consiste à différencier le cancer d'une pancréatite chronique. L'imagerie fonctionnelle (réalisée à l'aide de la tomographie par émission de positrons au fluorodésoxyglucose) peut s'avérer utile, surtout si les images sont fondues avec celles de la tomodensitométrie ou de l'IRM. La fiabilité diagnostique, les applications et les limites des diverses techniques d'imagerie font ici l'objet de discussion.
$\mathrm{P}^{\mathrm{a}}$ ancreatic cancer ranks among the five leading causes of death from cancer in adults in North America (1). The prognosis is very poor. The median survival time is approximately four months after diagnosis. Surgery is the only potential cure. Fewer than $3 \%$ of patients with pancreatic cancer are alive five years after diagnosis (2). In a series of 799 patients with a diagnosis of pancreatic adenocarcinoma, the five-year survival rate was $0 \%$ in patients who did not undergo resection compared with $24 \%$ in patients who underwent resection (3).

Abdominal pain (30\%), weight loss (36\%) and jaundice $(43 \%)$ are the main presenting symptoms (4). For the majority of patients, however, these symptoms occur late in the course of disease, and by the time the patient presents to the physician, the disease has progressed beyond the stage at which surgery is likely to be curative. A diagnosis is more likely to be made at an earlier stage of disease in patients presenting with jaundice than in those presenting with weight loss and abdominal pain. Nevertheless, more than $85 \%$ of pancreatic adenocarcinomas have extended beyond the pancreas at the time of diagnosis (5).

If there is to be any improvement in the dismal prognosis associated with this disease, it is essential that it be diagnosed early in its course. Neoplasms smaller than $1 \mathrm{~cm}$ in diameter have the best five-year prognosis, whereas lesions that are larger than $1.1 \mathrm{~cm}$ in diameter have a significantly poorer outlook (6). The challenge for imaging, therefore, is to detect these small lesions reliably.

Abdominal ultrasound (US), helical computed tomography (CT), magnetic resonance imaging (MRI), endoscopic US and endoscopic retrograde cholangiopancreatography have emerged as the main imaging modalities for the detection and staging of pancreatic cancer. These modalities rely on morphological changes in the pancreas for tumour detection and usually require the presence of at least two of the following observations to make a diagnosis: mass effect, altered attenuation/echogenicity/signal intensity, dilated pancreatic duct and/or pancreatic atrophy proximal to the tumour, and tumour extension beyond the gland to encase the regional vessels.
There are two main drawbacks to these modalities - lack of sensitivity for small (less than $2 \mathrm{~cm}$ in diameter) masses and the inability to differentiate tumour tissue from pancreatic masses caused by chronic pancreatitis (7). In addition, while these modalities are highly specific for determining nonresectability, they are not as good at predicting resectability because of difficulty in identifying metastatic lymphadenopathy, small liver metastases and small peritoneal implants.

Functional imaging with fluorodeoxyglucose positron emission tomography (FDG-PET) is evolving to complement and improve the sensitivity and specificity of the morphological modalities in detecting and staging pancreatic cancer.

\section{HELICAL CT}

CT is the most commonly used imaging modality for the detection and preoperative staging of pancreatic adenocarcinoma. CT is widely available, and the pancreas can be visualized in all patients. Pancreatic adenocarcinoma generally appears as a hypodense mass relative to the enhanced pancreatic parenchyma.

The recent introduction of multidetector row helical CT allows the acquisition of a larger volume of anatomy during any phase of intravenous contrast administration in a much shorter duration of time with no loss of image quality (8). More precise enhancement intervals are feasible, and thin collimated sections are routine. It is possible to retrospectively reconstruct the raw CT data into images that are thinner than the original set of reconstructed images, which produces improved anatomical detail. These images can be reformatted into off-axis planes to produce angiographic images and unique anatomical displays along the plane of the pancreas or pancreatic duct that were not previously possible (9). In a recent article, McNulty et al (10) showed that it is easiest to see the tumour during either the pancreatic parenchymal phase or the portal venous phase of contrast enhancement. These two phases are also associated with maximum opacification of the celiac and superior mesenteric arteries (pancreatic phase), and of the superior mesen- 
teric and portal veins (portal venous phase). Combining these two phases in the same study maximizes the chances of detecting direct tumour involvement of these vessels.

Overall, CT has a positive predictive value for tumour detection of greater than $90 \%(11-14)$. CT is also excellent for determining nonresectability, with a positive predictive value approaching $100 \%(11-14)$. It is not as accurate at identifying resectable tumours. The negative predictive value is $79 \%$ with dual-phase, or biphasic, helical CT (14). CT is poor at detecting small peritoneal implants, small hepatic surface and parenchymal metastases, and lymph node metastases in normal sized nodes $(13,15)$.

Technological advances will continue to improve CT in the future, including the development of faster scanners.

\section{US}

US is the most widely available cross-sectional imaging modality. It is inexpensive and does not use ionizing radiation. It is not always possible to visualize the pancreas well with US because of overlying bowel gas. With experience and diligence, however, it is possible to visualize the pancreas adequately in most patients. Techniques include the use of different windows, various respiratory manoeuvres and different patient positions. Oral contrast agents may also be employed to improve visualization of the pancreas, although these are not widely used in clinical practice.

Adenocarcinoma of the pancreas generally appears as an ill-defined, hypoechoic mass relative to the normal pancreas. US is excellent at assessing a dilated pancreatic duct and biliary tree, and its real-time capabilities often allow excellent assessment of the relationship of the tumour to these structures and to the regional vessels. US also facilitates an infinite number of imaging planes. Observations, such as the compliance of the superior mesenteric vein during compression and with changes in respiration, can be made in real time.

There is a wide variation in the published sensitivities of US in detecting pancreatic adenocarcinoma, suggesting that there are differences in technique. These differences likely reflect the experience and skill of the operators, and the time and effort spent visualizing the pancreas. At best, US has a sensitivity of $90 \%$ to $94 \%$ in detecting pancreatic cancer (16-18), but other investigators have reported sensitivities below 70\% (19-23).

Recent advances in US technology, such as harmonic imaging and compound imaging, may improve the sensitivity of US in this disease, but there are no published series to support this view.

\section{MRI}

In Canada, MRI is less available for pancreatic imaging than either US or CT. This will change as more MRI scanners are installed. Like US, MRI does not expose the patient to ionizing radiation, and technological advances over the recent years, including the introduction of breathheld gradient-echo sequences, have greatly improved image quality of the pancreas.
Pancreatic MRI can be performed with conventional spin-echo pulse sequences, with breath-held gradient-echo sequences using intravenous gadolinium contrast enhancement, or with $\mathrm{T} 1$-weighted sequences using manganesedipyridoxyl diphosphate (24). A T1-weighted spin-echo sequence with fat suppression offers significant advantages for imaging the pancreas compared with other noncontrastenhanced pulse sequences (25). This pulse sequence produces a high signal to noise ratio, reduces motion artifact with signal averaging, and is best for evaluating normal pancreatic anatomy, and differentiating between normal pancreas and pancreatic tumour. An adenocarcinoma appears as a low signal intensity mass relative to the normal pancreatic parenchyma. Breath-held gradient-echo images with gadolinium contrast enhancement are beneficial for detecting small tumours and liver metastases.

MRI has an accuracy similiar to that of CT for detecting and staging pancreatic adenocarcinoma, with published accuracies of $90 \%$ to $100 \%$ in series containing small numbers of patients $(26,27)$. MRI may have slightly better contrast resolution to distinguish the tumour from the pancreatic parenchyma, whereas CT may be slightly better at detecting vascular involvement. Thus, in certain circumstances, these tests may be complementary.

Magnetic resonance cholangiopancreatography is an excellent noninvasive method of obtaining images of the biliary and pancreatic ducts. These images can be displayed in an axial or three-dimensional format. The findings of pancreatic cancer and chronic pancreatitis obtained with magnetic resonance cholangiopancreatography are identical to those obtained with endoscopic retrograde cholangiopancreatography.

\section{FDG-PET}

Current clinical experience with FDG-PET in the detection of pancreatic cancer is limited to a few major centres. FDG-PET imaging is based on the observation that malignant tissues have a higher uptake of FDG compared with normal tissue. FDG is taken up by malignant cells in exactly the same way as glucose. Once inside the cell, it is not metabolized and, therefore, accumulates over time.

Initial investigation into the utility of PET in the diagnosis of pancreatic cancer began in the early 1990s. Initial reports suggested a sensitivity of $80 \%$, a specificity of $90 \%$ and an overall accuracy of $82 \%$ (28). Higashi et al (29) prospectively evaluated 54 patients with suspected pancreatic tumours and found an overall accuracy of $93 \%$ in successfully differentiating benign from malignant pancreatic disease. Shreve (30) has shown, however, that false-positive PET images can be produced by inflammatory pancreatic masses.

There are conflicting reports as to the utility of FDG$\mathrm{PET}$ in the identification and localization of regional lymph node metastases and small liver metastases.

Patients should be scanned after a 6 to $12 \mathrm{~h}$ fast. They should be adequately hydrated and should not be physically active. Patients with diabetes should be euglycemic. Even 
still, false negative FDG-PET results may occur in patients with insulin-dependent diabetes mellitus, because of altered FDG distribution.

It is likely that the greatest value of FDG-PET occurs with image fusion with either CT or MRI, thus combining the morphological detail of CT and MRI with the functional information from FDG-PET. It is hoped that this will not only improve the detection of small pancreatic tumours, but also help to distinguish malignant from inflammatory masses in the pancreas, and metastatic from reactive lymph nodes in the peripancreatic regions.

\section{DISCUSSION}

What is the best image for early detection: CT, MRI, PET or US? There is no clear answer to this question. Rigid imaging protocols do not work well for the individual patient, and the imaging work-up for a patient suspected of having pancreatic cancer should be tailored to the individual. Screening a patient in a high risk population requires a different approach. Certain principles can be applied, however.

Cross-sectional imaging of the patient with jaundice should be performed before biliary stent placement, unless there is strong clinical evidence that the patient has stone disease rather than a tumour. Interpretation of CT, US and MRI is much more difficult once a biliary stent has been placed, and the sensitivity for detecting a small mass is reduced. In addition, imaging a patient before intervention allows for optimal treatment planning for that patient.

In a patient suspected of having pancreatic cancer, CT is a very reasonable first choice. It is widely available, can always visualize the pancreas, is sensitive at detecting the tumour and is accurate at predicting nonresectability. In patients with nonresectable disease, no further imaging is required, and all that remains is to obtain a biopsy to confirm the diagnosis. US is often a more efficient modality to guide the biopsy, but CT can be used in problematic patients in whom US guidance is not feasible. If the CT is equivocal regarding the presence of pancreatic cancer, vascular invasion or liver metastases, US in experienced hands is an excellent complementary test.

MRI, in place of CT, should be reserved for patients with a history of allergy to iodinated contrast agents or patients with a history of renal insufficiency. It may also be very helpful as a problem-solving tool when CT and US are unable to reach a diagnosis or define a tumour clearly.

FDG-PET is not widely available but will likely find its role as a complementary test to CT and MRI in an effort to improve detection of small pancreatic masses, to better stage pancreatic cancer (particularly lymph node involvement) and to help distinguish benign from malignant disease. Image fusion with CT and MRI may help direct the laparoscopic surgeon to biopsy suspect lymph nodes before proceeding to formal laparotomy.

Screening for early ductal adenocarcinoma of the pancreas in patients at high risk (hereditary pancreatitis, sporadic chronic pancreatitis) will likely fall into the realm of molecular markers. If imaging is performed, US is the best initial screening modality because it is readily available, does not expose the patient to ionizing radiation and has a high sensitivity for detecting pancreatic cancer when performed by an experienced operator.

\section{REFERENCES}

1. Boring CC, Squires TS. Cancer statistics, 1992. CA Cancer J Clin 1992;42:19-38.

2. National Cancer Institute. Annual cancer statistics review 1973-1988. NIH publication No. 91-1789. Bethesda: Department of Health and Human Services, 1991.

3. Geer RJ, Brennan MF. Prognostic indicators for survival after resection of pancreatic adenocarcinoma. Am J Surg 1993;165:68-73.

4. Bakkevold KE, Arnesjo B, Kambestad B. Carcinoma of the pancreas and papilla of Vater: presenting symptoms, signs and diagnosis related to stage and tumour site. A prospective multicentre trial in 472 patients. Scand J Gastroenterol 1992;27:317-25.

5. Haycox A, Lombard M, Neoptolemos J, Walley T. Review article: Current practice and future perspectives in detection and diagnosis of pancreatic cancer. Aliment Pharmacol Ther 1998;12:937-48.

6. Barkin JS, Goldstein JA. Diagnostic and therapeutic approach to pancreatic cancer. Biomed Pharmacother 2000;54:400-9.

7. Hosten N, Lemke AJ, Wiedenmann B, Bohmig M, Rosewicz S. Combined imaging techniques for pancreatic cancer. Lancet 2000;356:909-10.

8. Hu H, He D, Foley D, Fox SH. Four multidetector-row helical CT: image quality and volume coverage speed. Radiology 2000;215:55-62.

9. Johnson, CD. Pancreatic carcinoma: developing a protocol for multidetector row CT. Radiology 2001;220:3-4.

10. McNulty NJ, Francis IR, Platt JF, Cohan RH, Korobkin M, Gebremariam A. Multi-detector row helical CT of the pancreas: effect of contrast-enhanced multiphase imaging on enhancement of the pancreas, peripancreatic vasculature, and pancreatic adenocarcinoma. Radiology 2001;220:97-102.

11. Freeny PC, Marks WM, Ryan JA, Traverso LW. Pancreatic ductal adenocarcinoma: diagnosis and staging with dynamic CT. Radiology 1988;166:125-33.

12. Freeny PC, Traverso LW, Ryan JA. Diagnosis and staging of pancreatic adenocarcinoma with dynamic computed tomography. Am J Surg 1993;165:600-6.

13. Bluemke DA, Cameron JL, Hruban RH, et al. Potentially resectable pancreatic adenocarcinoma: spiral assessment with surgical and pathological correlation. Radiology 1995;197:381-5.

14. Diehl SJ, Lehmann KJ, Sadick M, et al. Pancreatic cancer: value of dual-phase helical CT in assessing resectability. Radiology 1998;206:373-8.

15. Tabuchi $\mathrm{T}$, Itoh $\mathrm{K}$, Ohshio $\mathrm{G}$, et al. Tumor staging of pancreatic adenocarcinoma using early and late-phase helical CT. AJR Am J Roentgenol 1999;173:375-80.

16. Lawson T. Sensitivity of pancreatic ultrasound in the detection of pancreatic disease. Radiology 1978;128:733-6.

17. Taylor KJW, Buchin PJ, Viscomi GN, Rosenfield AT. Ultrasonographic scanning of the pancreas. Radiology 1981;138:211-3.

18. Karlson BM, Ekbom A, Lindgren PG, Kallskog V, Rastad J. Abdominal US for diagnosis of pancreatic tumor: prospective cohort analysis. Radiology 1999;213:107-11.

19. Wanebo HJ, Vezeridis MP. Pancreatic carcinoma in perspective: a continuing challenge. Cancer 1996;78:580-91.

20. Pasanen PA, Partanen KP, Pikkarainen PH, Alhava EM, Janatuinen EK, Pirinen AE. A comparison of ultrasound, computed tomography and endoscopic retrograde cholangiopancreatography in the differential diagnosis of benign and malignant jaundice and cholestasis. Eur J Surg 1993;159:23-9.

21. Pasanen PA, Eskelinen M, Partanen K, Pikkarainen P, Penttila I, Alhava E. A prospective study of the value of imaging, serum markers 
and their combination in the diagnosis of pancreatic carcinoma in symptomatic patients. Anticancer Res 1992;12:2309-14.

22. Hessel SJ, Siegelman SS, McNeil BJ, et al. A prospective evaluation of computed tomography and ultrasound of the pancreas. Radiology 1982;143:129-33.

23. Pasanen P, Partanen K, Pikkarainen P, et al. Diagnostic accuracy of ultrasound, computed tomography and endoscopic retrograde cholangiography in the detection of pancreatic cancer in patients with jaundice or cholestasis. In Vivo 1992;6:297-301.

24. Mayo-Smith WW, Schima W, Saini S, Slater GJ, McFarland EG. Pancreatic enhancement and pulse sequence analysis using low-dose mangafodipir trisodium. AJR Am J Roentgenol 1998;170:649-52.

25. Winston CB, Mitchell DG, Outwater EK, Ehrlich SM. Pancreatic signal intensity of T1-weighted fat saturation images: clinical correlation. J Magn Reson Imaging 1995;5:267-71.

26. Ichikawa T, Haradome H, Hachiya J, et al. Pancreatic ductal adenocarcinoma: preoperative assessment with helical CT versus dynamic MR imaging. Radiology 1997;202:655-62.

27. Smelka RC, Kroeker MA, Shoenut JP, Kroeker R, Yaffe CS, Micflikier AB. Pancreatic disease: prospective comparison of CT, ERCP and 1.5T MR imaging with gadolinium enhancement and fat suppression. Radiology 1991;181:785-91.

28. Bares R, Klever P, Hambuechen U, et al. Positron emission tomography (PET) with fluorine-18-labeled deoxyglucose (FDG) for the detection of pancreatic cancer: comparison with CT, ultrasonography (US), and ERCP. J Nucl Med 1993;34:98-108.

29. Higashi T, Tamaki N, Torizuka T, et al. Differentiation of malignant from benign pancreatic tumors by FDG- PET: comparison with CT, US, and endoscopic ultrasonography. J Nucl Med 1995;36:224-32.

30. Shreve PD. Focal fluorine-18 fluorodeoxyglucose accumulation in inflammatory pancreatic disease. Eur J Nucl Med $1998 ; 25: 259-64$ 


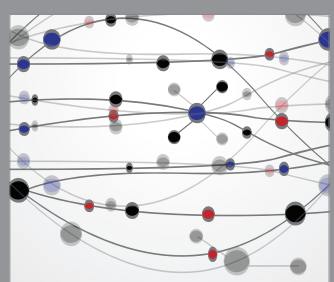

The Scientific World Journal
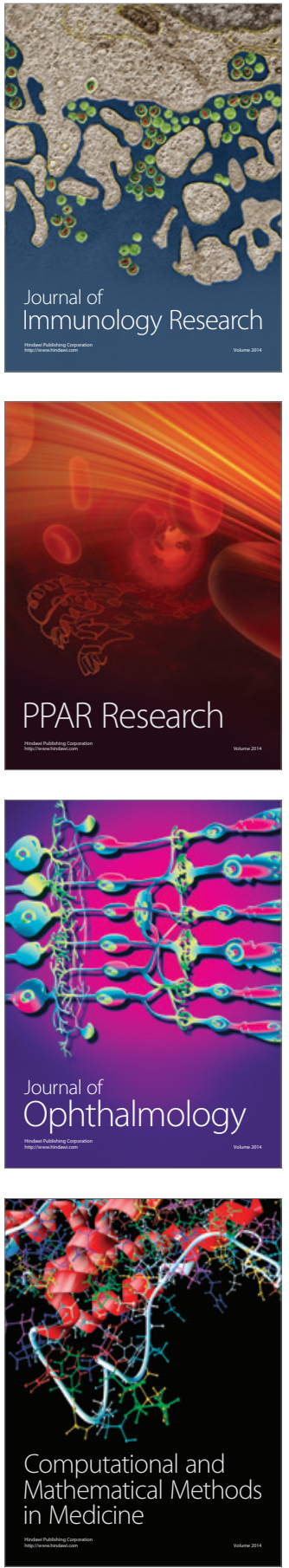

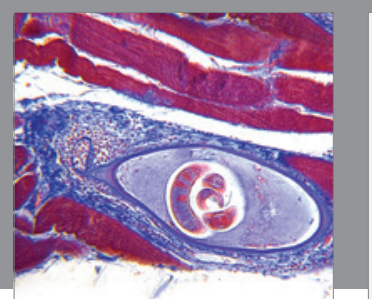

Gastroenterology Research and Practice

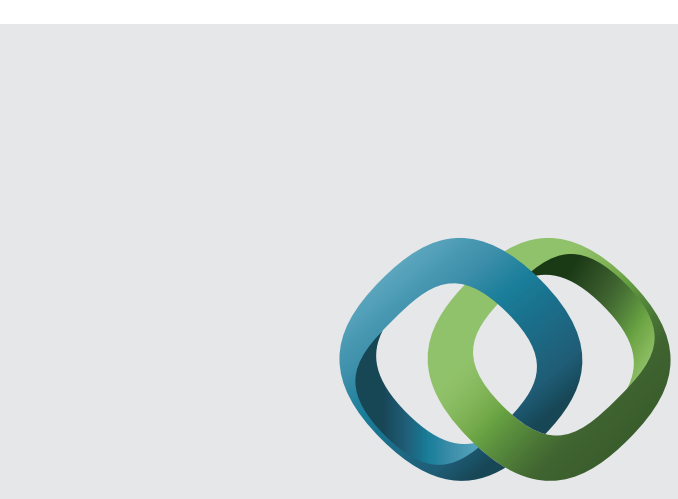

\section{Hindawi}

Submit your manuscripts at

http://www.hindawi.com
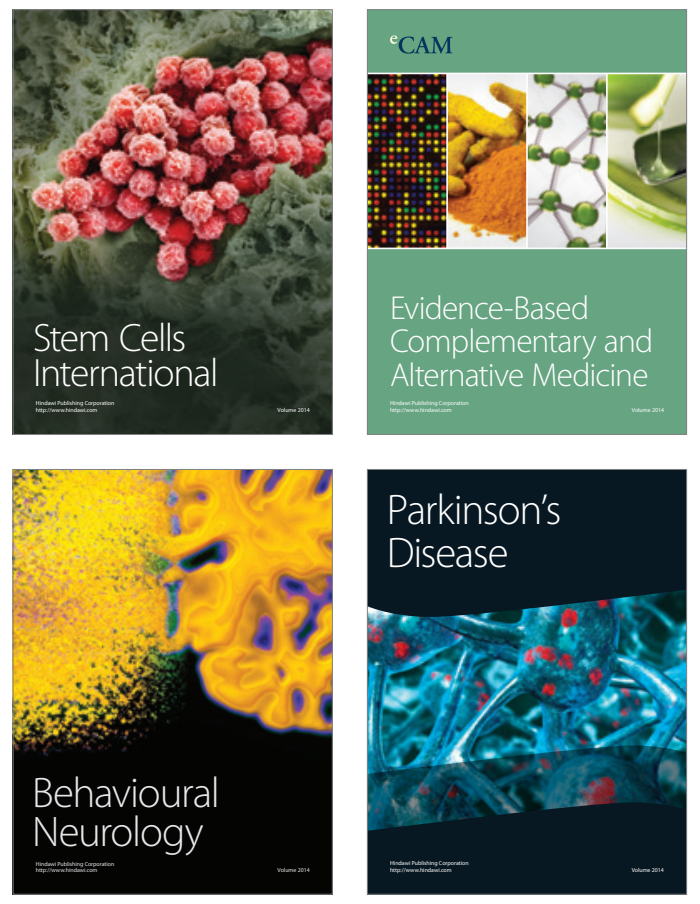
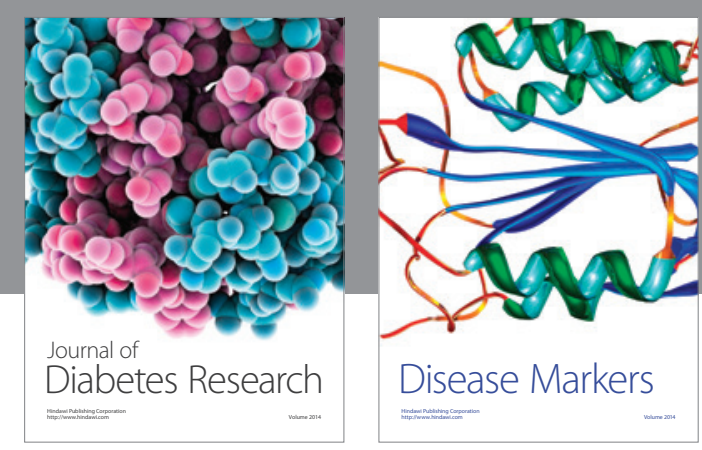

Disease Markers
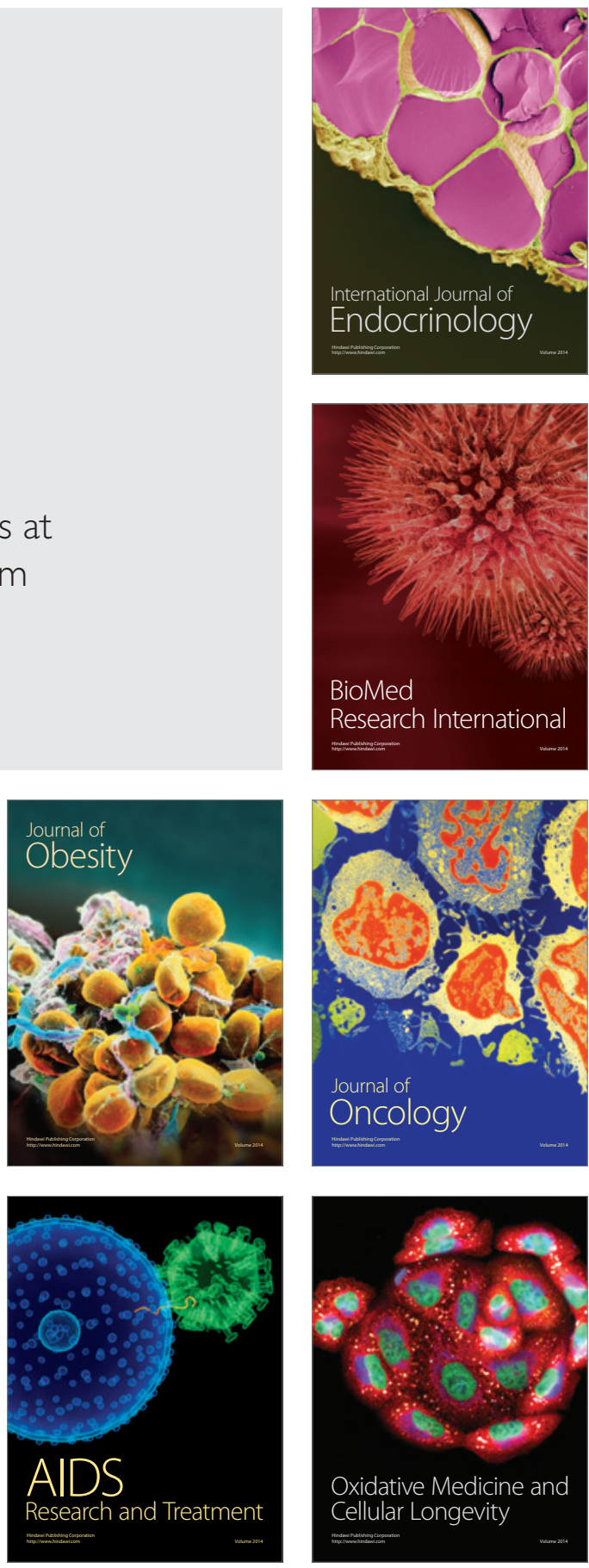